\title{
Survey on Patients' Perceived Satisfaction towards Dental Treatment Provided by Hospital Universiti Sains Malaysia (HUSM) Dental Clinic
}

\author{
Nadeerah binti Ibrahima, Ng Rou Enna, Adam Huseina* \\ School of Dental Sciences, Universiti Sains Malaysia, 16150 Kubang Kerian, Kelantan, Malaysia. \\ *E-mail: adamkck@usm.my
}

\begin{abstract}
Objective: Patients' perceived satisfaction towards dental treatment provided by HUSM Dental Clinic has never been reported before. This study aimed to evaluate patients' perceived satisfaction towards dental treatment provided by HUSM Dental Clinic and to investigate the factors influencing patients' perceived satisfaction regarding the treatment received besides collecting suggestions for improvement of dental services in HUSM Dental Clinic.

Methods: This is a cross-sectional study targeting dental patients who seek dental treatment in HUSM Dental Clinic.200 patients were interviewed by using a structured, interviewer-guided, adapted questionnaire.

Results: $48 \%$ of the subjects were between $18-25$ age group and 59.5\% of all subjects were earning <RM1000 per month. $78.5 \%$ of the respondents were satisfied with the dental treatment provided whereby $53 \%$ and $21.5 \%$ were satisfied and very satisfied respectively. $7.0 \%$ of the respondents were dissatisfied. Dental specialists, dental officers, post graduate students and undergraduate students produced $1.0 \%, 9.7 \%, 0 \%$ and $4.1 \%$ of dissatisfaction respectively. The major causes of satisfaction are friendly clinic staff (46.0\%), competent operator (39.5\%) and low treatment cost $(31.0 \%)$. As for patient dissatisfaction, the major causes are long waiting time (40.0\%), failure of operator to complete treatment on time (25.0\%) and poor service from clinic staff (15.0\%).
\end{abstract}

Conclusion: In general, majority of the respondents were satisfied with the treatment given to them. However, improvements are needed in some areas.

Keywords: dental treatment, patients' satisfaction

\section{Introduction}

Satisfying dental patients is a key task for all dental healthcare providers. Patients' satisfaction will also influence their compliance towards dental treatment indirectly and in turn the treatment outcome, especially for dental treatments that require multiple visits to the clinic in a specific period of time where patients' cooperation is crucial, such as orthodontic and endodontic treatment. ${ }^{1,2}$ Furthermore, dental patients nowadays are becoming increasingly concerned regarding the type of dental treatment received and the prognosis and outcome of the treatment itself. Therefore, it is very important for dental clinics to investigate and evaluate the level of patients' satisfaction towards dental treatment and services provided from time to time such that necessary measures can be carried out to improve the services provided, from the dental operator's technical skills to the facilities provided in the clinic to ensure that dental patients are satisfied. . $^{3,4}$
Majority of the studies on patients' satisfaction on dental treatment have started since 1980's, but most of the studies focused more on the roles of socio-demographic variables in determining patients' satisfaction towards dental treatment provided. ${ }^{5}$ There was very little empirical information available regarding consumers' acceptance of retail dental practices. ${ }^{6}$ Despite the consensus that patient satisfaction surveys are important in quality assurance in services and hospitals in the medical field, little work has been focused on patients' satisfaction with dental services.

The aim of this study was to evaluate patient's perceived satisfaction towards dental treatment provided by HUSM Dental Clinic. The specific objectives included, to investigate the factors influencing patients' satisfaction towards dental treatment provided in HUSM Dental Clinic and their relationship with patients' satisfaction, to identify the most influential factor in determining patients' satisfaction towards dental treatment provided in HUSM Dental Clinic and to investigate the roles of sociodemographic aspects in determining patients' satisfaction. 


\section{Methods and Materials}

A cross sectional study was conducted from the 28th of June 2015 to the 9th of July 2015, targeting 200 adult patients aged 18 years and above whom seeks dental treatment in HUSM Dental Clinic. Patients' satisfaction towards dental treatment provided by 5 different groups of dental operators, which are dental nurses, undergraduate dental students, postgraduate dental students, dental officers and dental specialists were investigated. A few factors were taken into consideration when evaluating dental patients' perceived satisfaction, such as sociodemographic factors, technical competency of dental operators, interpersonal factors, convenience in gaining access towards dental treatment in HUSM Dental Clinic, cost of treatment and the condition of the facilities provided. Structured, interviewer guided, adapted questionnaire was used in the data collection process..$^{7-9}$ Data collected was recorded and analyzed using the SPSS software version 22.0 which consists of transcript coding, detailed memo writing and data interpretation.

\section{Results}

200 respondents were interviewed for this research, consisting of $119(59.5 \%)$ females and $81(40.5 \%)$ males. Majority of the respondents were Malays (70\%), followed by Chinese $(25 \%)$, Indians $(1.5 \%)$ and other races such as Siamese and Kadazan (3.5\%).

Most of the respondents were from a young age group of $18-25$ years old with a percentage of $48 \%$ (96), followed by the age group of 36-40 years old with a percentage of $12.5 \%$ (25) and the age group of 26-35 years old with a percentage of $12 \%$ (24).

As for the education level, more than half of the respondents $(57.5 \%$ or 115$)$ were reported to have a higher education level which includes 25 (12.5\%) diploma holders, $85(42.5 \%)$ degree holders and $5(2.5 \%)$ are of postgraduate level. However, a vast majority of the respondents came from a lower income group where $57.5 \%$ or 115 of them were reported to have an income less than RM1000 per month. A smaller percentage which were $12.5 \%$ (25) of the respondents had an income of RM3000 and above per month. Only $2.0 \%$ of the respondents were reported to have no income.

Most of the respondents, $48.5 \%$ or 97 of them were treated by undergraduate students, followed by $31.0 \%$ (62) were treated by dental officers and $15.0 \%$ (30) were treated by dental specialists. Only a small percentage of respondents were treated by dental nurses and postgraduate student, with percentages of $1.3 \%$ (3) and $4.0 \%$ (8) respectively.

A majority of respondents were satisfied with the dental treatment provided by HUSM Dental Clinic. There were $57.5 \%$ (115) of the respondents who were satisfied and another $21.5 \%$ (43) of them were very satisfied. $14.0 \%$ or 28 of the respondents gave neutral responses, $6.5 \%$ of them said that they were dissatisfied and the remaining $0.5 \%$ or one of the respondents was very dissatisfied.

Furthermore, most of the respondents said that their overall experience upon seeking treatment in HUSM Dental Clinic was positive, with $52.0 \%$ or 104 of them saying that their overall experience was good and $13.5 \%$ or 27 of them answered "very good". $32.0 \%$ of them answered "satisfactory" and only $2.5 \%$ of them claimed to have bad overall experience in HUSM Dental Clinic.

The top 3 reasons which contribute to the respondents' satisfaction were friendly staff or operator (24.7\%), good technical competency of the dental operator when providing dental treatment $(21.2 \%)$ and low treatment cost $(16.7 \%)$. Other common reasons include complete clinical facilities (12.9\%), operator possessing excellent communication skills $(11.6 \%)$ and short waiting time $(9.4 \%)$.

As for those who were dissatisfied, most of them complained that the waiting time was too long, especially for those who were walk-in patients who needed urgent treatment. Other reasons are treatment cannot be completed on time, poor service from staff or operator and treatment failure.

$55.0 \%$ (110) of the respondents said that they usually waited less than 30 minutes before being called for treatment. $31.0 \%$ or 62 of them waited for $30-60$ minutes and $10.0 \%$ or 20 of them waited for $60-120$ minutes. Only $4 \%$ (8) of the respondents had to wait more than 120 minutes before their names were being called.

All respondents who were treated by postgraduate students were satisfied, with $75.0 \%$ of them reported to be satisfied and $25.0 \%$ of them being very satisfied. This is followed by the dental specialist group with a total satisfaction rate of $90.0 \%$, whereby $66.7 \%$ of the respondents were satisfied and $23.3 \%$ of them being very satisfied. As for the undergraduate students, there is a total satisfaction rate of $82.4 \%$.

The total satisfaction rate for dental officers is reported to be the lowest compared to the other groups, which is $66.1 \%$. In fact, the total dissatisfaction rate for dental officers is also the highest among the operator groups, which is $11.3 \%$, with $1.6 \%$ of the respondents being very dissatisfied and $9.7 \%$ being dissatisfied. The dental specialist group possesses the second highest total dissatisfaction rate, which is $10.0 \%$.

In general, the total satisfaction rate for male dental operators is slightly higher in comparison with that of female dental operators. The total satisfaction rate for male dental operators is $79.4 \%$ with $57.5 \%$ of the respondents being satisfied and $21.9 \%$ of them being very satisfied. As for female dental operators, the total satisfaction rate is $78.7 \%$, with $57.4 \%$ of the respondents being satisfied and $21.3 \%$ of them being very satisfied. On the other hand, the total dissatisfaction rate for female dental operators is higher, which is $7.9 \%$ in comparison 
with that of male dental operators, which is $5.5 \%$, whereby $1.4 \%$ of them being very dissatisfied and $4.1 \%$ of them being dissatisfied.

Pearson Chi Square test has been conducted to test the association between various socio-demographic variables and patients' perceived satisfaction towards dental treatment provided in HUSM Dental Clinic. As all the Pvalues obtained were $>0.05$, it is thus concluded that there is no significant association between all sociodemographic variables tested and patients' perceived satisfaction.

\section{Discussion}

It is revealed that $78.5 \%$ of the respondents were satisfied with the dental treatment provided, with $57.0 \%$ of them being satisfied and $21.5 \%$ of them being very satisfied. Only $6.5 \%$ were dissatisfied and $0.5 \%$ was very dissatisfied with the service provided. This shows that majority of the dental patients in HUSM Dental Clinic are satisfied with the dental treatment provided. However, as compared to a similar study done in the United Kingdom in year $2005,89.0 \%$ of the dental patients interviewed were satisfied. ${ }^{1,10}$ This means that, the satisfaction level of dental patients in HUSM Dental Clinic still needs improvement. The major causes for patient satisfaction are friendly staff or operator $(46.0 \%)$, technically competent operator $(39.5 \%)$ and low treatment cost $(31.0 \%)$. On the other hand, only a small portion of patients, $7.0 \%$ of them were dissatisfied, mostly caused by long waiting time before being called for treatment.

Most of the respondents, especially those who received treatment from undergraduate dental students claimed that they waited less than 30 minutes before they were called for dental treatment and there was actually $4.0 \%$ of them complained that they usually waited more than 120 minutes. This is because undergraduate dental students usually welcome their patients in right after they registered at the counter and those who waited long were usually walk-in patients due to limited dental officers and available treatment rooms at the outpatient clinic.

\section{Conclusion}

It is very important for the clinic administrations to hire more dental officers and specialists besides increasing the number of treatment rooms as suggested by some respondents. ${ }^{10}$ Furthermore, the efficiency of the counter service and improving the waiting list system should also be taken into consideration.

\section{Acknowledgment}

First and foremost, we will like to express our gratitude to our dental faculty, School of Dental Sciences, Universiti Sains Malaysia for providing us an opportunity to conduct this study as part of our elective course in Year 3 and 4.

\section{References}

1. R. Bedi, N. Gulati, C.McGrath, et al. A Study of satisfaction with dental services among adults in the United Kingdom. British DentalJournal. 1999;198 (7): 433-437

2. Alan Baldwin, Amrik Sohal, et al. Service quality factors and outcomes in dental care. AnInternational Journal. 2003;13 (3): 207-216.

3. Tin-Oo, et al (2011). Factors influencing patient satisfaction with dental appearance and treatments they desire to improve aesthetics.

4. P R H Newsome, G H Wright, et al. A review of patient satisfaction Dental patient satisfaction: an appraisal of recent literature: BritishDental Journal. 1999; 186: 166 . 170

5. Barnes, et al. Open wide: An examination of how patients select and evaluate their dentist. Health Market Q. 1985; 3: $49-56$.

6. Kress G, Silversin J, et al. The role of dental practice characteristics in patient satisfaction. GenDent. 1987; 35: 454-457.

7. Kress $\mathrm{G}$, et al. Improving patient satisfaction. Int Dent $\mathbf{J}$ 1987;37: 117-122.

8. Chakraborty G, Gaeth G, Cunningham $M$, et al Understanding consumers' preferences for dental service. J Health Care Market. 1993; 13: 48-58.

9. Andrus D, Buchheister J, et al (1985). Major factors affecting dental consumer satisfaction. HealthMarket Q 3 $57-68$.

10. Holt V, McHugh $\mathrm{K}$, et al. Factors influencing patient loyalty to dentist and dental practice. British Dental Journal. 1997; 183: 365-370. 